\title{
7th Scientific Meeting of the Japanese Society of Anti-Aging Medicine
}

\section{Contents}

My Meeting Report

Yoshikawa T, (Kyoto)

Selected Abstracts

Abstracts 1-14

Author Index 


\section{My Meeting Report}

The 7th Scientific Meeting of the Japanese Society of Anti-Aging Medicine organized by Prof Yoshikazu Yonei, Doshisha University, was held at the Kyoto International Conference Center (ICC), July 20-21, 2007.

The membership of this society has been increasing year by year and as of 2007 has surpassed 5,000 members. Over 2,000 participants attended the 7th Scientific Meeting, which is very high compared with other medical meetings - it consisted of one presidential lecture, two special lectures, two educational lectures, thirteen symposia, six workshops, ten satellite seminars, and more.

Because of limited space, it is difficult to introduce them all, but I would like to pick out some of the 'hot' topics.

Firstly, propellant of anti-aging by the anti-metabolic syndrome seems to be the most attractive one.

In related sessions, various topics not only to investigate the molecular biochemical analysis of the metabolic syndrome, but also to validate food factors and apply traditional medical treatment (Oriental medicine) and caloric restrictions became the important themes.

Although anti-aging medicine due to food factors is the most obvious field to study in Japan, there is still not enough scientific evidence for further discussion.

Especially, the presentation by Prof. Shirasawa of Juntendo University on the molecular mechanism of polyphenols for life extension gained a lot of attention from the participants. He showed that chronic treatment with polyphenols significantly extended longevity in mice.
Also the practical methods of the anti-aging medicine, especially the symposium on hormone therapies such as DHEA, growth hormone, melatonin, coenzyme Q, and estrogen, attracted large audiences.

Furthermore, another important topic that needs emphasis is anti-aging by exercise. Since many people worldwide exercise as part of their daily lives, scientific evaluation of the effectiveness of exercise is highly expected.

Finally, the various types of analytical methods that play an important role in evaluation of the individual and research into anti-aging medicine were introduced.

It seems that it will take a while to establish a scientific methodology to analyze anti-aging medicine, because various very important methodologies to evaluate the function of anti-oxidant foods are still being developed.

However, anti-ageing research in Japan is just beginning. The official meetings have only been held 7 times until now but, due to the participation of a lot of general physicians, knowledge in this field is spreading quickly.

Authorization as specialists and special institutions led by the Japanese Society of Anti-Aging Medicine have recently helped to broaden the scope of this field. I have the impression that anti-aging medicine will spread rapidly in Japan, and believe that the development of antiaging medicine will be very fruitful in the future.

Toshikazu Yoshikawa

\begin{tabular}{ll}
\hline KARGER & (c) 2007 S. Karger AG, Basel \\
$0250-6807 / 07 / 0515-0408 \$ 23.50 / 0$ \\
Fax +4161306 1234 \\
$\begin{array}{l}\text { E-Mail karger@karger.ch } \\
\text { www.karger.com }\end{array}$ & $\begin{array}{l}\text { Accessible online at: } \\
\text { www.karger.com/anm }\end{array}$
\end{tabular}


1

\section{Enhanced Glycolysis Is Common Metabolic Feature Observed in ES and Immortalized Primary Cells to Bypass Senescence}

\section{H. Kondoh \\ Department of Geriatric Medicine, Graduate School of Medicine, Kyoto University, Kyoto, Japan}

Aims: Enhanced glycolysis even less than $20 \%$ oxygen condition is the characteristic property of most cancer cells, called the Warburg effect. It is exploited clinically in detection of primary tumors and metastasis based on positron emission tomography measurement of 2-[18F] fluoro-2-deoxy-D-glucose uptake, suggesting undisputed significance of the Warburg effect in cancer. However, there have been few mechanistic insights into its relationship with the well-characterized molecular and genetic events associated with cellular immortalization.

Methods and Results: Through an unbiased screen for genes that can immortalize mouse embryonic fibroblasts (MEFs), we identified the glycolytic enzyme phosphoglycerate mutase (PGM). A twofold increase in PGM activity enhances glycolytic flux, allows indefinite proliferation of primary MEFs. Glucosephosphate isomerase (GPI), another glycolytic enzyme, displays similar activity and conversely depletion of PGM or GPI triggers premature senescence. Immortalized MEFs and mouse embryonic stem cells (ES cells) display higher glycolytic flux with reduced oxygen consumption, and more resistance to oxidative damage than senescent cells. These metabolic property might support the proliferative potential of ES cells, proven by the inhibition of its self-renewality or glycolytic flux. Finally we found molecular link between p53 axis and PGM. As wild-type p53 down-regulates PGM, mutation of p53 can facilitate immortalization via effects on PGM levels and glycolysis.

Conclusions: We discovered unexpected aspect of the Warburg effect, protecting cells from senescence effect of oxidative damage in primary cells and ES cells.

\section{2}

\section{The Expression Profile of Anti-Aging Related Genes in Dermal and Gingival Fibroblasts}

\author{
K. Ebisawa', R. Kato' ${ }^{2}$ T. Sugimura', H. Akimichi, \\ T. Katada ${ }^{3}$, Y. Narita ${ }^{1}$, H. Kagami ${ }^{4}$, M. Ueda $a^{1,4}$ \\ ${ }^{1}$ Nagoya University School of Medicine, ${ }^{2}$ Graduate School \\ of Engineering, Nagoya University, ${ }^{3}$ Reve21 Co., ${ }^{4}$ The \\ Institute of Medical Science, The University of Tokyo, Japan
}

Aims: With the growing attention to the field of regenerative medicine, cell therapy is considered one of the most promising clinical approaches for patients with various diseases. Cell therapy using fibroblasts has been applied in the treatment of wrinkles and scars. Fibroblasts are known to produce most of the extracellular matrices such as collagen and hyaluronic acid, and are therefore considered critical in skin rejuvenation. Since 2005, we have experienced more than 40 clinical cases of fibroblast injection for facial wrinkles and scar treatment and have observed improvements. However, the mechanism of this approach has not been studied in detail. To investigate the gene expression profile of cultured fibroblasts harvested from the dermis and gingiva by microarray analysis.

Methods: Dermal and gingival fibroblasts were harvested from healthy volunteers from whom informed consent was obtained according to the protocol approved by the ethics committee of Nagoya University Hospital. Cells were cultured and expanded in Dulbecco's modified Eagle's medium containing fetal bovine serum. The cultured cells between passages 3 and 4 were harvested for total mRNA extraction. The gene expression data were obtained using Human Genome Focus Array (Affymetrix), and analyzed by Arrayassist (Stratagene).

Results: From the comparison of expression profiles of dermal and gingival fibroblasts among 5,284 genes, 5.3\% of genes showed a significant difference in the level of expression. However, 19 genes that related to anti-aging activity were more highly expressed in the gingival fibroblasts than those in the dermal fibroblast with significance $(\mathrm{p}<0.05, \mathrm{FC}>2.0)$.

Conclusions: Our results indicate that the fundamental characters of both dermal and gingival fibroblasts are almost identical; however, gingival fibroblast treatment might be beneficial for skin rejuvenation, not only by extracelluar matrix production, but also by the expression of these unique anti-aging related genes.

3

\section{'Super-Microsurgery' for Ovarian Transplantation and Vascularized Whole Organ Cryopreservation, and for Lady's Lifecycle Preservation}

\author{
M. Mihara', M. Narushima1', I. Koshima', S. Tainosho² \\ ${ }^{1}$ Department of Plastic and Reconstructive Surgery, Tokyo \\ University, ${ }^{2}$ Department of anatomy, Tokyo Medical \\ University, Tokyo, Japan
}

Aims: Chemotherapy and radiotherapy are essential for treatment of cancer in children and young adults, but these methods often result in testicular failure or reduced ovarian function. To date, priority has been given to the cancer therapy and the side effects have largely been disregarded; however, research into embryo cryopreservation may alter this balance. This procedure might be performed before cancer therapy and, in particular, may offer hope for preservation of fertility in young women with cancer. One problem in this area

\begin{tabular}{ll}
\hline KARGER & C 2007 S. Karger AG, Basel \\
0250-6807/07/0515-0409\$23.50/0 \\
$\begin{array}{l}\text { Fax +4161306 1234 } \\
\text { E-Mail karger@karger.ch } \\
\text { www.karger.com }\end{array}$ & $\begin{array}{l}\text { Accessible online at: } \\
\text { www.karger.com/anm }\end{array}$
\end{tabular}


is that the ovum is structurally weak, compared with sperm, and the pregnancy rate after frozen ovum storage is very low. We hypothesize that this is due to instability of blood flow, and to examine this possibility we have focused on the vascular system and cryopreservation using a rat ovary organ transplantation model.

Methods and Results: The basis of our work is a technology which we refer to as 'super-microsurgery'. Microvascular anastomosis under a microscope has been used for about 10 years in plastic surgery, and use of 12-0 nylon string (needle: $50 \mathrm{~mm}$ ) allows for an anastomosis of about $0.3 \mathrm{~mm}$. Plastic surgeons commonly use soft tissue implantation as a free flap for patients with carcinoma and trauma. We are examining the potential of this technology in transplantation and cryopreservation of the ovary, with preservation not only of the shredding tissue but of the vascularized whole organ. We attempted transplantation of a vascularized ovary following a certain period of cryopreservation. After this period, the ovary was defrosted and the ovarian blood vessel was anastomosed to a subcutaneous blood vessel anywhere in the body. The endocrine function of the ovary and the potential for pregnancy were permanently reacquired following this procedure, which required 'super-microsurgery' because the ovarian blood vessel in rat is $0.3-0.5 \mathrm{~mm}$ in diameter.

Conclusions: This method can enrich lady's lifecycle.

\section{4}

\section{Analysis of Model Mice for Heart Failure Using Cre-loxP System}

S. Kawakami1,2,3, T. Shimizu1,2,3, H. Nojiri1,4, M. Funakoshi',
T. Shirasawa1,4
${ }^{1}$ Research Team for Molecular Biomarker, Tokyo
Metropolitan Institute of Gerontology, ${ }^{2}$ Anti-Aging Science
Inc., ${ }^{3}$ United Graduate School of Agricultural Science,
Tokyo University of Agriculture and Technology, ${ }^{4}$ Juntendo
University School of Medicine, Tokyo, Japan

Aims: Elderly people with the symptoms of chronic heart failure are impaired in quality of life. Currently, the morbidity and mortality rate increase in an age-dependent manner. Almost all of the heart failure is dysfunction of left ventricular (LV) performance. A substantial number of animal model studies have shown that reactive oxygen species (ROS) play a pathological role in failing heart. However, the molecular mechanism of the pathogenesis is not clear. Manganese superoxide dismutase (Mn-SOD) is a principal scavenger enzyme located in mitochondrial matrix, which detoxifies superoxide $\left(\mathrm{O}_{2}^{-}\right)$by dismutation, protecting cells from oxidative stress.

Methods and Results: In order to investigate pathological role a long-term oxidative injury in the heart, we generated heart/musclespecific Mn-SOD deficient mice (H/M-Sod2 $2^{--}$) using Cre-loxP system. H/M-Sod $2^{-1-}$ pups were born with the expected mendelian ratio and grown without morphological abnormalities. However, by 6 months after birth, the mutant mice were dead. H/M-Sod2 ${ }^{-/-}$mice showed marked enlargement of heart mass compared with control mice. In echocardiography, H/M-Sod2 $2^{-/-}$mice exhibited dilated cardiomyopathy, as evidenced by the marked dilated LV cavity and lower ejection fraction ( 2 months old, $21 \% ; 4$ months old, 16\%).

In order to evaluate daily physical activity of mutant mice, we placed a running wheel apparatus in their cages. They showed low level of spontaneous motor activity. Moreover, to study the relationship between cardiac dysfunction and mitochondrial function, we assessed mitochondrial respiratory activity. The result showed that the respiratory activity and ATP production were significantly reduced in the heart of H/M-Sod2 $2^{-/}$mice. Furthermore, H/M-Sod $2^{-/-}$mice also showed enhanced generation of $\mathrm{O}_{2}^{-}$in heart mitochondria.

Conclusions: We showed here that the mitochondrial oxidative stress caused heart failure associated with impairment of mitochondrial respiration and ATP production. We presented a murine model of heart/muscle-specific aging and devised a strategy for preventive medicine with therapeutic treatment for age-related or oxidative stress-dependent heart disease.

\section{5}

\section{The Role of Oxidative Stress in Resistance to Ischemia/Reperfusion Injury and Refractoriness to Ischemic Preconditioning in Aged Heart}

\author{
K. Yamashita1, H. Otani ${ }^{1}$, T. Okazaki', A. Moriguchi', \\ D. Sato', S. Matsuhisa', Y. Akita', C. Enoki², H. Imamura², \\ T. Iwasaka \\ ${ }^{1}$ The Second Department of Internal Medicine, Division of \\ Cardiology, ${ }^{2}$ The Department of Thoracic and \\ Cardiovascular Surgery, Kansai Medical University, \\ Moriguchi, Japan
}

Aims: Aged hearts are known to be refractory to ischemic preconditioning (IPC). It has been demonstrated that aged hearts are exposed to oxidative stress by activation of angiotensin II type 1 receptors (AT1R). Because IPC is triggered by oxidative stress, we hypothesized that aged hearts are preconditioned to ischemia/reperfusion (I/R) injury by oxidative stress and are not further protected by IPC but removal of oxidative stress by blockade of AT1R restores IPC in these hearts.

Methods: Young (10 weeks old) and aged (24-30 months old) rats were treated with an antioxidant, N-acethylcysteine (NAC; $1 \mathrm{~g} / \mathrm{kg} /$ day, p.o.) or an AT1R blocker, losartan ( $30 \mathrm{mg} / \mathrm{kg} /$ day, p.o.) for 2 weeks. These hearts were excised, buffer-perfused, and subjected to 30 minutes ischemia followed by 2 hours reperfusion. Ischemic preconditioning (IPC) was performed by 3 cycles of 5 minutes ischemia and 5 minutes reperfusion before 30 minutes ischemia.

Results: Infarct size was not significantly different between young and aged hearts ( $40 \pm 3 \%$ vs. $42 \pm 3 \%$ ). Nitrotyrosine formation as an index of oxidative stress was increased in aged hearts and inhibited by NAC and losartan. NAC and losartan had no effect on infarct size in young hearts but the former drug significantly $(p<0.05)$ increased infarct size in aged hearts $(55 \pm 6 \%)$. IPC reduced infarct size in young hearts $(18 \pm 2 \%)$ but not in aged hearts $(39 \pm 3 \%)$. NAC abolished the infarct size-limiting effect of IPC in young hearts ( $34 \pm 4 \%$ ) but had no effect on IPC in aged hearts (46 $\pm 5 \%$ ). In contrast, losartan did not block IPC in young hearts $(16 \pm 1 \%)$ and restored the infarct sizelimiting effect of IPC in aged hearts $(19 \pm 2 \%)$.

Conclusions: These results suggest that aged hearts are exposed to oxidative stress by activation of AT1R which plays a role in protection against $\mathrm{I} / \mathrm{R}$ injury and refractoriness to IPC and that removal of oxidative stress by AT1R blockers but not genuine antioxidants restores cardioprotection by IPC in these hearts. 


\section{6}

\section{Heme Oxygenase-1 Prevents Vascular Senescence through Mechanisms Involving SIRT1/ p53/p21 Pathway in Aged-Mouse}

E. Terada ${ }^{1}$, B. Saji1 ${ }^{1}$ G. Yoshino ${ }^{2}$, J. Yamasaki³, T. Morita ${ }^{4}$

${ }^{1}$ Department of Pediatrics, ${ }^{2}$ Division of Diabetes, Metabolism and Endocrinology, Department of Internal

Medicine, ${ }^{3}$ Division of Cardiovascular Medicine,

Department of Internal Medicine, ${ }^{4}$ Department of

Laboratory Medicine, Toho University School of Medicine, Tokyo, Japan

Aims: In yeast, restriction of calorie extends life span by increasing activity of Sir2, an NAD+-dependent deacetylase. SIRT1, a human homolog of Sir2, has been reported to inhibit p53, which is one of the key players in stimulating cellular senescence in vascular wall. Heme oxygenase is a microsomal enzyme that catalyzes the degradation of heme into biliverdin, which is subsequently reduced to bilirubin, free iron and carbon monoxide (CO). Induction of heme oxygenase-1 (HO-1) has been potentially associated with cellular protection, especially against oxidative insults, which promote agerelated changes.

Methods and Results: In this study, using smooth muscle celldirected HO-1 over-expression mice (HO-1 Tg), we investigated antiaging effect of HO-1 on cardiovascular system in aged mouse. Fifty weeks old wild type mouse (Wt) displays enhanced in vivo cellular senescence as determined by senescence-associated beta-gal staining as well as increased expression of p53 and p 21 in the aorta. Consistent with these findings, expression of SIRT1 was markedly decreased, while inflammatory cytokines were increased and eNOS was decreased, respectively, in the aorta of Wt. In contrast, there are no significant changes in these senescence-related markers, and expression of SIRT1 was not reduced in age-matched HO-1 Tg.

Conclusions: Taken together, these findings indicate that vascular HO-1 counteracts vascular senescence through restoration of SIRT1 as well as inhibition of p53/p21 pathway in aorta. Vascular HO-1 can be a new therapeutic target against age-related cardiovascular diseases.

7

Preventive Effect of Resveratrol, a Red Wine Polyphenol, on the Rotenone-Induced Cytotoxicity in the Cellular Model of Parkinson's Disease

\section{S. Kyuhou}

Department of Physiology, Kansai Medical University, Moriguchi, Japan

Aims: Parkinson's disease (PD) is reported to be caused by an interaction between genetic and environmental factors. Fewer studies clarified environmental factors. Rotenone, a pesticide, is recently highlighted because rotenone treatment to animals produces many features of PD including behavioral abnormalities, selective degeneration of nigral dopaminergic neurons and formation of ubiquitin-positive aggregates in nigral neurons. Since rotenone was reported to inhibit the complex I of the electron transport chain and produces reactive oxygen species (ROS), resveratrol, an antioxidant polyphenol included in red wine, is expected to prevent the toxicity of rotenone.

Methods: PC12 cells were cultured in the medium including nerve growth factor. Cell viability was evaluated with tetrazolium salt. Total RNA was extracted from the PC12 cells and gene expression was measured by real-time RT-PCR. Generation of ROS was detected with dichlorodihydrofluorescein diacetate.

Results: Rotenone induced PC12 cell to die in concentration dependent manner. Degenerating PC12 cells were stained by terminal deoxynucleotidyl transferase mediated dUTP nick end labeling and anti-active caspase 3 antibody, indicating that the degenerating PC12 undergo cell death via apoptosis. Prominent production of ROS was observed after rotenone exposure. The endoplasmic reticulum (ER) stress were found to be involved in this rotenone-induced apoptosis because up-regulation of CHOP and activation of Jun-N-terminal kinase, ER stress activated substrates, were observed. Up-regulation of mRNA of BiP, an ER-specific chaperone, and splicing of the mRNA of the $X$ box binding protein were observed, indicating the occurrence of the unfolded protein response (UPR). Preincubation of resveratrol reduced the rotenone-induced ROS and UPR. The viability of PC12 cells was significantly increased by resveratrol.

Conclusions: Resveratrol protected PC12 cells from the cytotoxicity of rotenone, suggesting its use as a preventive agent for PD.

\section{8}

Impact of a Smoking Habit on Perimenopausal Bone Mineral Density
K. Fujii', A. Higo ${ }^{1}$, H. Shibata ${ }^{2}$, H. Hirose ${ }^{1,2}$, T. Moriki, ${ }^{1,2}$, M. Tsujioka ${ }^{1,2}$, S. Nanri ${ }^{1}$, I. Saito ${ }^{1,2}$
${ }^{1}$ Department of Internal Medicine, ${ }^{2}$ Health Center, Keio University School of Medicine, Tokyo, Japan

Aims: Estrogen concentration is lowered at menopause, leading to the activation of osteoclasts and loss of bone mineral density (BMD). Diet, exercise, cessation of smoking, and moderation of alcohol consumption are effective in preventing loss of BMD at this stage of life. It is a concern that many postmenopausal smoking women try to control BMD loss by increasing calcium intake without smoking cessation. We compared the impact of smoking and other life-style factors on perimenopausal BMD in female university employees.

Methods: Of 489 women whose BMD was measured in an annual physical examination in 2006, 31 (age: $54 \pm 3$ years old) had experienced menopause three to five years before the examination. We retrospectively evaluated the changes in their calcaneal BMD from 2001 to 2005 and conducted 2-group comparisons, by dividing these 31 women based on smoking habit, alcohol consumption, exercise habit and dietary calcium intake. T-score (\%) was used as an index of BMD which was measured using an ultrasonic bone evaluation device (AOS-100, Aloka, Tokyo). Life-style factors were surveyed using a self-report questionnaire, and calcium intake was calculated using Excel Eiyo-kun FFQg (Kenpakusha). The MannWhitney U-test was used in the two-group comparisons and $\mathrm{p}<0.05$ was considered significant. The same analysis was conducted on 
other 66 women (age: $50 \pm 2$ years old) with regular menstruation as a control.

Results and Conclusions: Loss of BMD after menopause was observed in all 31 women. The subjects with a smoking habit $(\mathrm{n}=7)$ suffered significantly greater BMD loss $(p=0.0264)$ than those without a smoking habit $(n=24)$. There was no significant difference in loss of BMD between groups divided by their alcohol consumption, exercise habit or calcium intake. Because smoking women with regular menstruation did not demonstrate similar tendency, we speculated that menopause increased the sensitivity to smoking habit, resulting in greater loss of BMD. Our results suggest that smoking cessation is most important among life-style moderations to prevent loss of BMD in perimenopausal women.

\section{9}

\section{Oral Zinc Supplementation Inhibits Hepatic Fibrosis by Reducing TIMP-1 Activity}

\author{
M. Takahashi,2, H. Saito ${ }^{1}$, M. Higashimoto ${ }^{3}$, T. Hibi ${ }^{1}$ \\ ${ }^{1}$ Department of Internal Medicine, School of Medicine, \\ Keio University, ${ }^{2}$ Department of Gastroenterology, \\ National Tokyo Medical Center, ${ }^{3}$ Department of Clinical \\ Laboratory, Hiro-o Metropolitan Hospital, Tokyo, Japan
}

Aims: It has been reported that serum and hepatic zinc concentrations are decreased in patients with chronic liver diseases and zinc supplementation shows favorable effects on liver fibrosis in vivo and in vitro. The mechanism of antifibrotic effect of zinc supplementation has not been understood.

Methods: The informed consent was given to patients with early liver cirrhosis (Child-Pugh A) (zinc supplied; $\mathrm{n}=17$ ) and the other 5 patients (control group; $n=5$ ). Oral zinc supplementation was given with polaprezinc (a Japanese medicine for protection of gastric mucosae), for 24 weeks ( $34 \mathrm{mg} /$ day zinc), and various markers of liver fibrosis, and fibrogenic and fibrolytic enzyme activities were monitored. Patients having polaprezinc were further divided into two categories: patients whose serum zinc levels increased by more than $1 \mathrm{SD}$ above the baseline after 24 weeks (group A; $\mathrm{n}=5$ ); and patients whose serum zinc levels did not increase or increased less than 1 SD (group B; $\mathrm{n}=7$ ).

Results: Pretreatment serum zinc concentration was shortly reduced in all patients $(52.5 \pm 11.4 \mu \mathrm{g} / \mathrm{dl}$ [range: $32-74])$. No side effects were noted in all patients. Oral polaprezinc administration increased serum zinc concentrations of 17 zinc-supplied subjects by $31.6 \%(\mathrm{p}<0.01)$ at 24 weeks, while no change was noted in the control group. The increase in serum zinc levels in the group A patients was $55.9 \%(p<0.05)$ at 24 weeks. Serum zinc levels did not change in patients of the control and group B at the end of study. In the group A patients, the serum levels of type IV collagen and the TIMP-1 activity were significantly reduced, but no such change was observed in other groups, and no other serum markers changed.

Conclusions: These results suggest that oral zinc supplementation may be a useful strategy for anti-fibrosis therapy in patients with early liver cirrhosis.
10

\section{Gender and Age Related Differences on Serum Amino Acid among Healthy Japanese Subjects}

N. Kasezawa, M. Niizato, N. Ohsugi, K. Tohyama, M. Shimbo, H. Yokogoshi

Shizuoka Medical Center, Saitama Prefectural University, Department of Health Sciences, and University of Shizuoka, School of Food and Nutritional Sciences, Shizuoka, Japan

Aims: Amino acids provide the principal substrate necessary for protein synthesis and they also serve wide range of physiological function. Various factors that might influence serum amino acid concentration in the normal health individual have been investigated worldwide, but information on the serum amino acid among Japanese people is sparse. The objectives of the present study were to examine if gender and age per se affects serum amino acid concentration in Japanese people.

Method: A cross-sectional study was performed in April 2004 to March 2005. The serum amino acids have been analyzed in 777 healthy subjects without treatment and taking medicine (male/female: 463/314, age range: $25-84$ years old). The height, weight, body mass index (BMI), biochemical variables and serum amino acid profiles were evaluated.

Results: The mean age of subjects in male and female was 51.6 and 47.7 years old, respectively. The mean serum albumin and BMI values in the subjects were in normal range. Compared with female, higher essential amino acids for example branch chain amino acid (BCAA) and the most of nonessential amino acids were observed in male. However serum glycine and serine were higher in female than male. The changes of serum valine, isoleucine, BCAA, methionine, tryptophan, asparagines and aspartic acid with aging were not observed in both genders. The serum lysine, tyrosine, arginine, glutamic acid, ornithine, citrulline and aromatic amino acid increased with age in both genders. On the other hand, serum alpha - amino butyric acid and Fischer's ratio decreased with age. The concentration in serum threonine and cysteine decreased with age in the elderly subjects (more than 65 years old).

Conclusions: The age and gender related differences in serum amino acid concentrations. Glycine and serine were higher in female than in male. Amino acid which participates in urea cycle of metabolism increased with age in both genders. 
11

\section{Novel Nutritional Markers for Care-Risk Assessment in Elderly People - Plasma Concentrations of Amino Acids and Redox Status of Serum Albumin}

\author{
T. Shimizu 1,5, T. Muramatsu2, T. Ando2, T. Takehana ${ }^{3}$, \\ S. Obuchi ${ }^{4}$, T. Shirasawa ${ }^{1,5}$ \\ ${ }^{1}$ Research Team for Molecular Biomarkers, Tokyo \\ Metropolitan Institute of Gerontology, ${ }^{2}$ Institute of Life \\ Science, AJINOMOTO CO., INC., ${ }^{3}$ Pharmaceutical \\ Research Laboratories, AJINOMOTO CO., INC, \\ ${ }^{4}$ Department for Prevention of Dependence on Long-term \\ Care, ${ }^{5}$ Anti-Aging Science, INC., Tokyo, Japan
}

Aims: Health condition may affect the metabolism in elderly people. It is well known that metabolic abnormalities are often occurred to the patients with malnutrition, renal function or inflammation, in plasma concentrations of amino acids and ratio of serum mercaptalbumin to total serum albumin (= mercaptalbumin + nonmercaptalbumin). The objective was to clarify the novel nutritional makers for the care-risk assessment in the elderly. We chose plasma concentrations of amino acids and ratio of serum mercaptalbumin for evaluation as markers.

Methods: 145 elderly subjects in elderly nursing home (59 males and 86 females, age: $80.9 \pm 6.9$ years old) were analyzed for plasma concentrations of amino acids and the ratio of serum mercaptalbumin. Mini Nutritional Assessment (MNA)-test was performed for each subject.

Results: From the MNA-tests, 5 subjects with malnourished (MNA score $<17), 51$ at risk of malnutrition $($ MNA score $=$ 17-23.5) and 89 with no malnutrition (healthy control, MNA score $>23.5$ ) were obtained. Plasma essential amino acid concentration was significant lower in subjects at risk of malnutrition than in healthy control, although the concentrations of serum albumin and non essential amino acids were not changed. The ratio of serum mercaptalbumin was significantly inverse-correlated with age $(\mathrm{p}<0.001)$, serum creatinine $(\mathrm{p}<0.0001)$ and serum $\mathrm{C}$-reactive protein concentrations $(\mathrm{p}<0.0001)$.

Conclusions: Plasma concentration of essential amino acids is useful for assessing the risk of malnutrition in the elderly, whose serum albumin concentration is still not changed. The ratio of serum mercaptalbumin was related to aging, and allows evaluating the renal function and inflammation in elderly for nursing care.
12

\section{Usability of Nail-Based Microelement Analysis in Anti-Aging Medicine and Development of Nail-Based Gene Analysis}

\author{
T. Katsuya, S. Kobayashi, K. Sugimoto, H. Rakugi, \\ T. Ogihara \\ Department of Geriatric Medicine, Osaka University \\ Graduate School of Medicine, Osaka, Laboratory of \\ Nutritional Biochemistry, Graduate School of agricultural \\ and Life Sciences, The University of Tokyo, Osaka General \\ Medical Center, Osaka Prefectural Organization, Tokyo, \\ Japan
}

Aims: To pursue the anti-aging medicine, supplementation of minerals or vitamins is now popular all over the world. However, there was no appropriate indicator for medium term effect of the diet, supplementation or medication. To identify new indicator of medium term of internal environment in anti-aging medicine, we carried out nail microelement analysis using hypertensives and healthy volunteers. In addition, we developed nail-based gene analysis system.

Methods: Hypertensive patients $(\mathrm{n}=192)$ were recruited form outpatients of Osaka University Hospital and hypertension was defined that SBP/DBP was above $140 / 90 \mathrm{mmHg}$ or chronically under antihypertensive medication. Healthy volunteers $(n=1,096)$ were recruited throughout Japan. We measured 17 nail microelements using inductively coupled plasma mass spectrometer, ICPM-8500 (Shimadzu Inc.). To develop nail-based gene analysis, we examined the optimal proteolysis condition of keratins without the activation of nuclease. The study protocol was approved by Ethical Committee of Osaka University.

Results: Along with aging, $\mathrm{Mg}, \mathrm{Zn}, \mathrm{Cr}, \mathrm{Mn}, \mathrm{Al}, \mathrm{Hg}, \mathrm{As}$ and $\mathrm{Cd}$ levels were significantly increased whereas $\mathrm{Na}$ and $\mathrm{K}$ levels were decreased. In males, $\mathrm{P}, \mathrm{Na}, \mathrm{K}, \mathrm{Mg}, \mathrm{Pb}, \mathrm{Hg}$ and As levels were significantly higher than that in females but $\mathrm{Zn}$ and $\mathrm{Al}$ levels were higher in females. After adjustment of age, sex and body mass index, Na and $\mathrm{K}$ levels in nail was significantly $(\mathrm{p}<0.007)$ higher in hypertensives than that in healthy volunteers. We also succeeded to extract the enough amount of DNA from the nails to be able to detect the gene polymorphisms.

Conclusions: Nail-based microelement or gene analysis is useful for the evaluation of early stage of life related disease or medium term effect of diet, supplementation or medication.

\section{3 \\ Estimation of Optimal Vitamin D Status \\ M. Nakao', S. Nakano², T. Mitsuo ${ }^{1}$ \\ ${ }^{1}$ Mitsuo Clinic, Tokyo, ${ }^{2}$ Sagamihara Chuou Hospital, Sagamihara, Japan}

Aims: Vitamin D has been regarded as an important determinate for bone health. Alternatively, vitamin D protects both malignant diseases such as colorectal cancer, breast cancer and prostate cancer, and 
autoimmune diseases including multiple sclerosis, rheumatoid arthritis and scleroderma. The concentration of serum vitamin D (25-hydroxy vitamin D) is reduced by aging or improper nutritional status. Vitamin $\mathrm{D}$ is an essential nutrition for human well-being; however, there is no consensus on optimal levels of the serum 25-hydroxyvitamin D. We addressed the dose of vitamin D (vitamin D3) required to achieve or maintain optimal serum 25-hydroxyvitamin D concentration.

Methods: The subjects were 173 men and 197 women of our clinic patients. At the first visit, serum 25 -hydroxyvitamin D concentrations of them were measured. If the patients had a level of 25hydroxyvitamin D less than $20 \mathrm{ng} / \mathrm{ml}$, we administrated $1000 \mathrm{IU}$ of vitamin D3 to the patients, and disclosed the changes of the serum vitamin D concentration.

Results: There were 44 men (25\%) and 83 women (42\%) that the serum concentration of 25-hydroxyvitamin D was less than $20 \mathrm{ng} / \mathrm{ml}$. After administration of 1000 IU of vitamin D3, the mean serum 25hydroxyvitamin D raised from $17.1 \pm 2.6$ to $24.5 \pm 4.5 \mathrm{ng} / \mathrm{ml}$ in men and from $15.0 \pm 2.6$ to $29.2 \pm 8.0 \mathrm{ng} / \mathrm{ml}$ in women. There was no adverse effect such hypercalcemia during this therapy.

Conclusions: The levels of serum 25-hydroxyvitamin D of the patients that the one was less than $20 \mathrm{ng} / \mathrm{ml}$ were raised to optimal levels after administration of 1000 IU of vitamin D3.

14

\section{Evaluation of Intima-Media Thickness of the Common Carotid Artery with Natural Longevity in Western Region in China}

Y. Kasamaki1, M. Ohta', A. Shindo', T. Nakayamai², Y. Izumii ${ }^{2}$, H.K. Matsumoto ${ }^{2}$, H. Shimabukuro', T. Ueki, M. Yoshida ${ }^{3}$, E. Kobayashi1, A. Hirayama1', S. Saito1, S. Nakagawa ${ }^{5}$, Y. Ozawa ${ }^{1}$

${ }^{1}$ Division of Cardiology, Nihon University School of Medicine, 2 Division of Nephrology and Endocrinology, Nihon University School of Medicine, ${ }^{3}$ Department of Dentistry, Nihon University School of Medicine, ${ }^{4}$ Division of Genomic Epidemiology and Clinical trials, Nihon University School of Medicine, ${ }^{5}$ Nihon University School of Medicine, Tokyo, Japan

Aims: The Hotan region in Xinjiang, China, is famous for the natural longevity of the Uygur people (UL). According to our conventional results, the total cholesterol (TC), low-density lipoprotein cholesterol (LDL-C), triglyceride (TG) and fasting glucose in UL were lower than in other ethnic groups. Therefore, we hypothesized that the development of arteriosclerosis would be slow in the UL. The aim of this study was to clarify the relationship between aging and intima-media thickness (IMT) of the common carotid artery (CCA).

Methods: We used ultrasonography (ACUSON Cypress, $7 \mathrm{MHz}$ electronic linear probe, Siemens) to study the carotid arteries of 113 subjects over 90 years old. IMT of the CCA was measured. Blood pressure was evaluated by $24-\mathrm{h}$ ambulatory blood pressure monitoring. Hypertention was defined as an systolic blood pressure (SBP) $>160 \mathrm{mmHg}$ and/or a diastolic blood pressure (DBP) $>95 \mathrm{mmHg}$.

Results: IMT of the CCA in the UL was lower value $(0.85 \pm 0.14 \mathrm{~mm})$ when compared with a value supposed by their age. Aging was not correlated with IMT $\left(\mathrm{r}^{2}=0.0003\right)$ in subject populations. Prevalence of hypertention was $31.4 \%$. SBP and DBP were $122 \pm 15,70 \pm 7 \mathrm{mmHg}$, respectively. Aging was less correlated with systolic blood pressure $(\mathrm{r}=0.27, \mathrm{p}<0.05)$.

Conclusions: Our results suggest that less IMT associated with the slower development of arteriosclerosis in the UL could be attributed to natural longevity. 


\section{Nateition Metabolism}

Numbers refer to abstract numbers
Akimichi, H. 2

Akita, Y. 5

Ando, T. 11

Ebisawa, K. 2

Enoki, C. 5

Fujii, K. 8

Funakoshi, M. 4

Hibi, T. 9

Higashimoto, M. 9

Higo, A. 8

Hirayama, A. 14

Hirose, H. 8

Imamura, H. 5

Iwasaka, T. 5

Izumii, Y. 14

Kagami, H. 2

Kasamaki, Y. 14

Kasezawa, N. 10
Katada, T. 2

Kato, R. 2

Katsuya, T. 12

Kawakami, S. 4

Kobayashi, E. 14

Kobayashi, S. 12

Kondoh, H. 1

Koshima, I. 3

Kyuhou, S. 7

Matsuhisa, S. 5

Matsumoto, H.K. 14

Mihara, M. 3

Mitsuo, T. 13

Moriguchi, A. 5

Moriki, T. 8

Morita, T. 6

Muramatsu, T. 11

Nakagawa, S. 14

Nakano, S. 13

Nakao, M. 13

Nakayamai, T. 14
Nanri, S. 8

Narita, Y. 2

Narushima, M. 3

Niizato, M. 10

Nojiri, H. 4

Obuchi, S. 11

Ogihara, T. 12

Ohsugi, N. 10

Ohta, M. 14

Okazaki, T. 5

Otani, H. 5

Ozawa, Y. 14

Rakugi, H. 12

Saito, H. 9

Saito, I. 8

Saito, S. 14

Saji, B. 6

Sato, D. 5

Shibata, H. 8

Shimabukuro, H. 14
Shimbo, M. 10

Shimizu, T. 4, 11

Shindo, A. 14

Shirasawa, T. 4, 11

Sugimoto, K. 12

Sugimura, T. 2

Tainosho, S. 3

Takahashi, M. 9

Takehana, T. 11

Terada, E. 6

Tohyama, K. 10

Tsujioka, M. 8

Ueda, M. 2

Ueki, T. 14

Yamasaki, J. 6

Yamashita, K. 5

Yokogoshi, H. 10

Yoshida, M. 14

Yoshino, G. 6 